\title{
High Redshift Lyman Limit and Damped Lyman-Alpha Absorbers
}

\author{
L.J. Storrie-Lombardi ${ }^{1,2}$, R.G. McMahon ${ }^{1,5}$, M.J. $\operatorname{Irwin}^{3}$, C. Hazard ${ }^{1,4}$ \\ ${ }^{1}$ Institute of Astronomy, Madingley Road, Cambridge CB3 OHA UK \\ 2 current address: UCSD-CASS, Mail Code 0111, La Jolla, CA 92093 USA lsl@ucsd.edu \\ ${ }^{3}$ Royal Greenwich Observatory, Madingley Road, Cambridge CB3 0HE UK mike@ast.cam.ac.uk \\ ${ }^{4}$ University of Pittsburgh, Pittsburgh, PA USA \\ ${ }^{5}$ rgm@ast.cam.ac.uk
}

To appear in "ESO Workshop on QSO Absorption Lines" Preprint: astro-ph/yymmddd

\begin{abstract}
We have obtained high signal:to:noise optical spectroscopy at $5 \AA$ resolution of 27 quasars from the APM z>4 quasar survey. The spectra have been analyzed to create new samples of high redshift Lyman-limit and damped Lyman- $\alpha$ absorbers. These data have been combined with published data sets in a study of the redshift evolution and the column density distribution function for absorbers with $\log \mathrm{N}(\mathrm{HI}) \geq 17.5$, over the redshift range $0.01<\mathrm{z}<5$. The main results are:

- Lyman limit systems: The data are well fit by a power law $N(z)=N_{0}(1+z)^{\gamma}$ for the number density per unit redshift. For the first time intrinsic evolution is detected in the product of the absorption cross-section and comoving spatial number density for an $\Omega=1$ Universe. We find $\gamma=1.55$ ( $\gamma=0.5$ for no evolution) and $N_{0}=0.27$ with $>99.7 \%$ confidence limits for $\gamma$ of $0.82 \& 2.37$.

- Damped Ly $\alpha$ systems: The APM QSOs provide a substantial increase in the redshift path available for damped surveys for $z>3$. Eleven candidate and three confirmed damped Ly $\alpha$ absorption systems, have been identified in the APM QSO spectra covering the redshift range $2.8 \leq z \leq 4.4$ (11 with $z>3.5$ ). Combining the APM survey confirmed and candidate damped Ly $\alpha$ absorbers with previous surveys, we find evidence for a turnover at $\mathrm{z} \sim 3$ or a flattening at $\mathrm{z} \sim 2$ in the cosmological mass density of neutral gas, $\Omega_{g}$.
\end{abstract}

The Lyman limit survey results are published in Storrie-Lombardi, et al., 1994, ApJ, 427, L13. Here we describe the results for the DLA population of absorbers.

\section{Introduction}

How and when galaxies formed are questions at the forefront of work in observational cosmology. Absorption systems detected in quasar spectra provide the means to study these phenomena up to z 5 , back to when the Universe was less than $10 \%$ of its present age. While the baryonic content of spiral galaxies that are observed in the present epoch is concentrated in stars, in the past this must have been in the form of gas. Damped Ly $\alpha$ absorption (DLA) systems have neutral hydrogen column densities of $\mathrm{N}(\mathrm{HI})>2 \times 10^{20} \mathrm{~cm}^{-2}$. They dominate the baryonic mass contributed by HI. The principal gaseous component in spirals is HI which 
has led to surveys for absorption systems detected by the DLA they produce (Wolfe, Turnshek, Smith \& Cohen 1986 [WTSC]; Lanzetta et al. 1991 [LWTLMH]; Lanzetta, Wolfe \& Turnshek 1995 [LWT95]). We extend the earlier work on Lyman limit systems and DLAs to higher redshifts using observations of QSOs from the APM z>4 QSO survey (Irwin, McMahon \& Hazard 1991), These data more than triple the redshift path surveyed at $\mathrm{z}>3$ and allow the first systematic study up to $\mathrm{z}=4.5$.

\section{$2 \quad$ APM Damped Lyman-Alpha Survey at $\mathrm{z} \sim 4$}

We have obtained 27 high $\mathrm{S} / \mathrm{N}$ spectra at $5 \AA$ resolution at the William Herschel Telescope. The spectra were analyzed starting $3000 \mathrm{~km} \mathrm{~s}^{-1}$ blueward of $\mathrm{z}$ (emission). The analysis was stopped when the $\mathrm{S} / \mathrm{N}$ ratio became too low to detect a Ly $\alpha$ line with $\mathrm{W}$ (rest) $\geq 5 \AA$. This point was typically caused by the incidence of a Lyman limit system. Features with $\mathrm{W}$ (observed) $>25 \AA$ were selected with an automated procedure. Most of these are blends of the dense Ly $\alpha$ forest features present at high redshift. The equivalent width and FWHM were measured interactively and $\mathrm{N}(\mathrm{HI})$ was estimated for features with $\mathrm{W}$ or FWHM $>30 \AA$. Of the 34 measured, 15 have estimated $\mathrm{N}(\mathrm{HI}) \geq 2 \times 10^{20} \mathrm{~cm}^{-2}$ covering $2.8 \leq z \leq 4.4$. Only one candidate has estimated $\mathrm{N}(\mathrm{HI}) \geq 10^{21} \mathrm{~cm}^{-2}$. High resolution spectroscopy of 4 candidates has confirmed 3 as damped $(\log \mathrm{N}(\mathrm{HI}) \geq 20.3)$. The sensitivity of the survey with redshift was determined using the method developed by Lanzetta (see LWT95). The function $g(z)$ is calculated, giving the number of lines of sight along which a damped system at a redshift $z$ could be detected. Figure 1a shows the sensitivity of the APM survey alone and in combination with the WTSC and LWTLMH surveys.

\section{Evolution of the Number Density per Unit Redshift}

The candidate and confirmed DLA systems from the APM sample and previous surveys (WTSC; LWTLMH; LWT95) have been combined to study the evolution of the number density per unit redshift for $0.01<\mathrm{z}<4.7$. Fit with the customary power law $N(z)=$ $N_{0}(1+z)^{\gamma}$, a population with no intrinsic evolution in the product of the absorption crosssection and comoving spatial number density will have $\gamma=1 / 2(\Omega=1)$ or $\gamma=1(\Omega=0)$. A maximum likelihood fit to the data with $\mathrm{z}>1.5$ yields $N(z)=0.03(1+z)^{1.5 \pm 0.6}$, consistent with no intrinsic evolution even though the value of $\gamma$ is similar to that found for the Lyman limit systems where evolution is detected at a significant level. However, there is redshift evolution evident in the higher column density systems with an apparent decline in $N(z)$ for $z>3.5$. These results are displayed in figure 1(b). The combined data set is plotted as dashed lines with the above fit. The results for only the absorbers with $\log \mathrm{N}(\mathrm{HI}) \geq 21$ are shown as solid lines. The $\mathrm{z}<1.5$ bin is taken from LWT95. 


\section{Evolution of $\Omega_{g}-$ Baryons in Neutral Gas}

The mean cosmological mass density contributed by damped Ly $\alpha$ absorbers can be estimated as

$$
\left\langle\Omega_{g}\right\rangle=\frac{H_{0} \mu m_{H}}{c \rho_{\text {crit }}} \int_{N_{\text {min }}}^{\infty} N f(N) d N
$$

as defined in LWTLMH (equations 17-18), giving the current mass density in units of the current critical density. The errors in $\Omega_{g}$ are difficult to estimate because the column density distribution function, $f(N)$, is not known. LWTLMH utilised the standard error in the distribution of $\mathrm{N}(\mathrm{HI})$ which yields zero error if all the column densities in a bin are the same. We have estimated the fractional variance in $\Omega_{g}$ as $\sum_{i=1}^{p} N_{i}^{2} /\left(\sum_{i=1}^{p} N_{i}\right)^{2}$ which yields $\sqrt{n}$ errors if all the column densities included in a bin are equal. This method yields larger errors. The results for $\Omega_{g}$ are shown in figure 2 for $\mathrm{q}_{0}=0$ and $\mathrm{q}_{0}=0.5\left(\mathrm{H}_{0}=50\right)$. The $\mathrm{z}<1.5$ bin is taken from LWT95. The $\mathrm{z}>1.5$ solid bins utilise the data from WTSC, LWTLMH, and the APM survey. The dotted bins exclude the APM data. The inclusion of the APM survey data for $z>3$ lowers the value previously found for $3<z<3.5$ and indicates a possible turnover for $z>3.5$. The results are also consistent with a relatively constant value of $\Omega_{g}$ for $z>2$ as the error bars are still very large at high redshift. Larger samples of bright $z>4$ quasars are needed.

\section{Summary}

The QSOs from the APM survey more than triple the $z>3$ redshift path for DLA surveys. Fourteen candidate DLA systems have been identified in the APM spectra covering $2.8 \leq$ $z \leq 4.4$ (11 with $z>3.5$ ), with 3 confirmed. Combining these data with the previous surveys and fitting a single power law for $\mathrm{z}>1.5$ gives $\mathrm{N}(\mathrm{z})=.03(1+z)^{1.5 \pm 0.6}$, marginally consistent with no evolution models. Evolution is evident in the highest column density absorbers with the incidence of systems with $\log \mathrm{N}(\mathrm{HI}) \geq 21$ apparently decreasing for $\mathrm{z}_{\sim}^{>} 3.5$. We find evidence for a turnover or flattening in the cosmological mass density of neutral gas, $\Omega_{g}$ at high redshift. The more gradual evolution of $\Omega_{g}$ than previously found helps alleviate the 'cosmic G-dwarf problem' (LWT95), i.e. if a large amount of star formation has taken place between $\mathrm{z}=3.5$ and $\mathrm{z}=2$, a much larger percentage of low metallicity stars should exist than is detected. It is also consistent with the suggestion by Pettini et al. (1994) that the wide range in DLA metallicities measured at the same epoch indicates that at $\mathrm{z} \sim 2$ they are observed prior to the bulk of star formation in the disk. 


\section{References}

[1] Gnedin, N.Y. \& Ostriker, J.P., 1992, ApJ, 400,1

[2] Irwin, M.J., McMahon, R.G. \& Hazard, C., 1991, in ASP Conf. Series, Vol. 21, ed. Crampton D., (San Francisco: ASP), 117

[3] Lanzetta, K.M., Wolfe, A.M., Turnshek, D.A., Lu, L., McMahon, R.G. \& Hazard, C., 1991, ApJS, 77, 1 (LTWLMH)

[4] Lanzetta, K.M., Wolfe, A.M. \& Turnshek, D.A., 1995, ApJ, 440, 435 (LWT95)

[5] Pettini, M., Smith, L.J., Hunstead, R.W. \& King, D.L., 1994, ApJ, 426, 79

[6] Rao, S. \& Briggs, F., 1993, ApJ, 419, 515

[7] Storrie-Lombardi, L.J., McMahon, R.G., Irwin, M.J. \& Hazard 1994, ApJ, 427, L13

[8] Wolfe, A.M., Turnshek, D.A., Smith, H.E. \& Cohen R.D., 1986, ApJS, 61, 249 (WTSC)

\section{Figure Captions}

Figure 1: (a) The sensitivity function, $g(z)$, of the DLA surveys. This gives the number of lines of sight along which a damped system at redshift $z$ could be detected. (b) The number density of DLA per unit redshift, $N(z)$, vs. $\mathrm{z}$ (absorption). The dashed bins show $\mathrm{N}(\mathrm{z})$ for all the damped systems and the solid bins for systems with $\mathrm{N}(\mathrm{HI}) \geq 10^{21} \mathrm{~cm}^{-2}$. A single power law fit to the sample for $\mathrm{z}>1.5$ gives $\mathrm{N}(\mathrm{z})=.03(1+z)^{1.5 \pm 0.6}$.

Figure 2: The mean cosmological mass density in neutral gas, $\Omega_{g}$, contributed by DLA absorbers for $0.01 \leq \mathrm{z} \leq 4.7$ for $\mathrm{q}_{0}=0$ and $\mathrm{q}_{0}=0.5\left(\mathrm{H}_{0}=50\right)$. The $\mathrm{z}<1.5$ bin is taken from LWT95. The $z>1.5$ solid bins utilise the WTSC, LWTLMH, and APM survey data. The dotted bins exclude the APM data. The points at $\mathrm{z}=0$ are $\Omega_{\text {stars }}$ (star) from Gnedin \& Ostriker (1992) and $\Omega_{H I}$ (circle) from Rao \& Briggs (1993). 


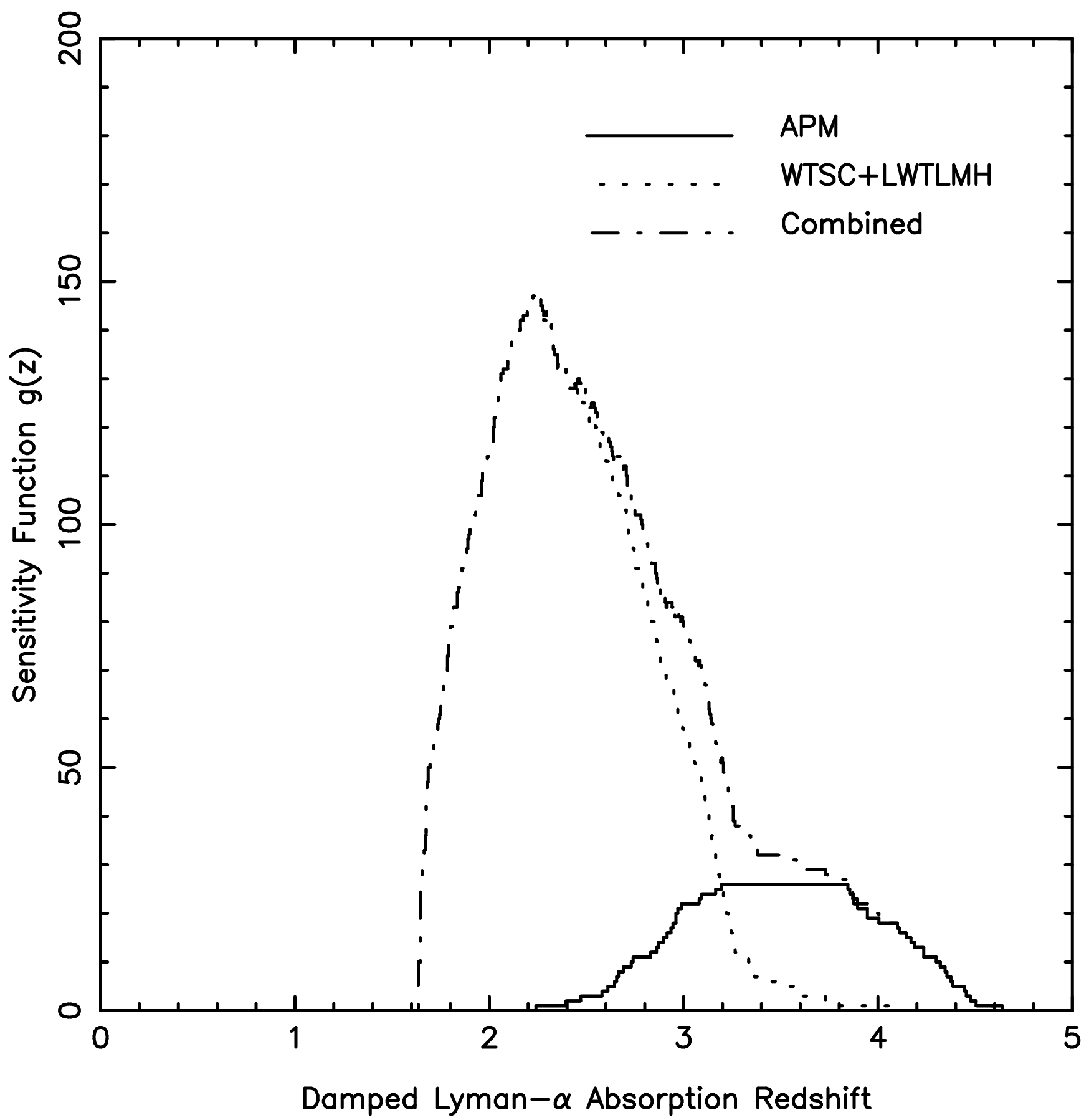




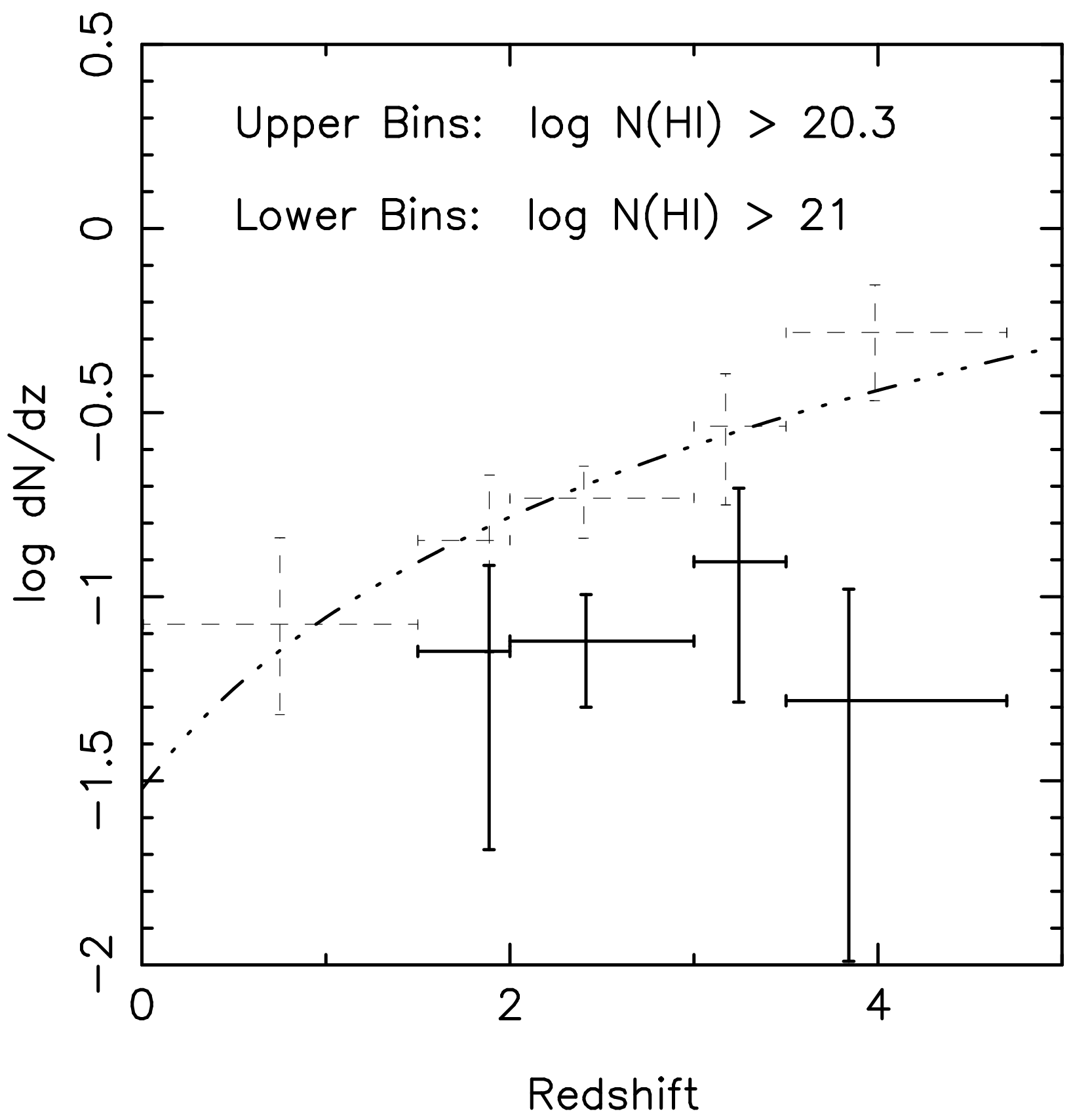




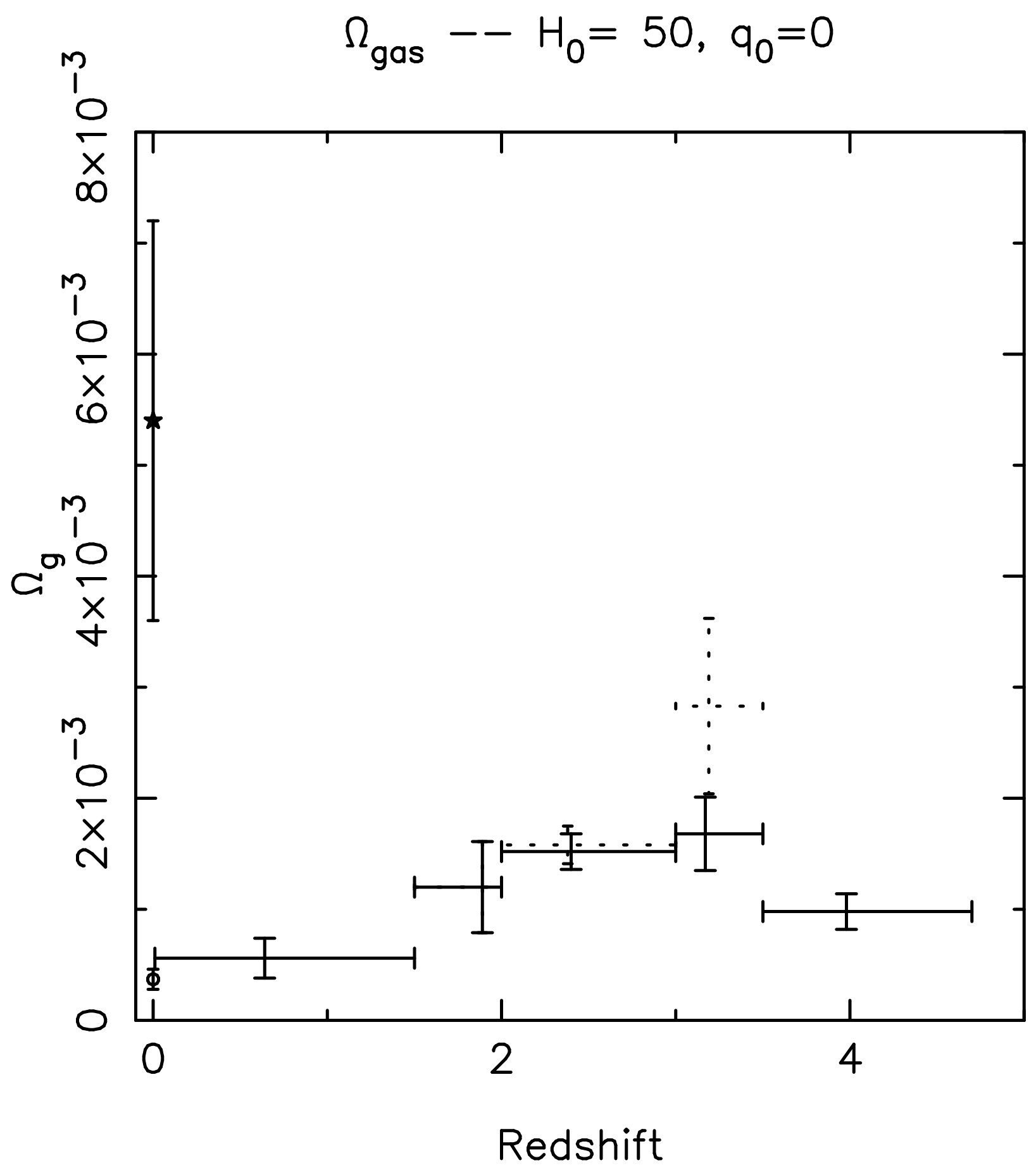




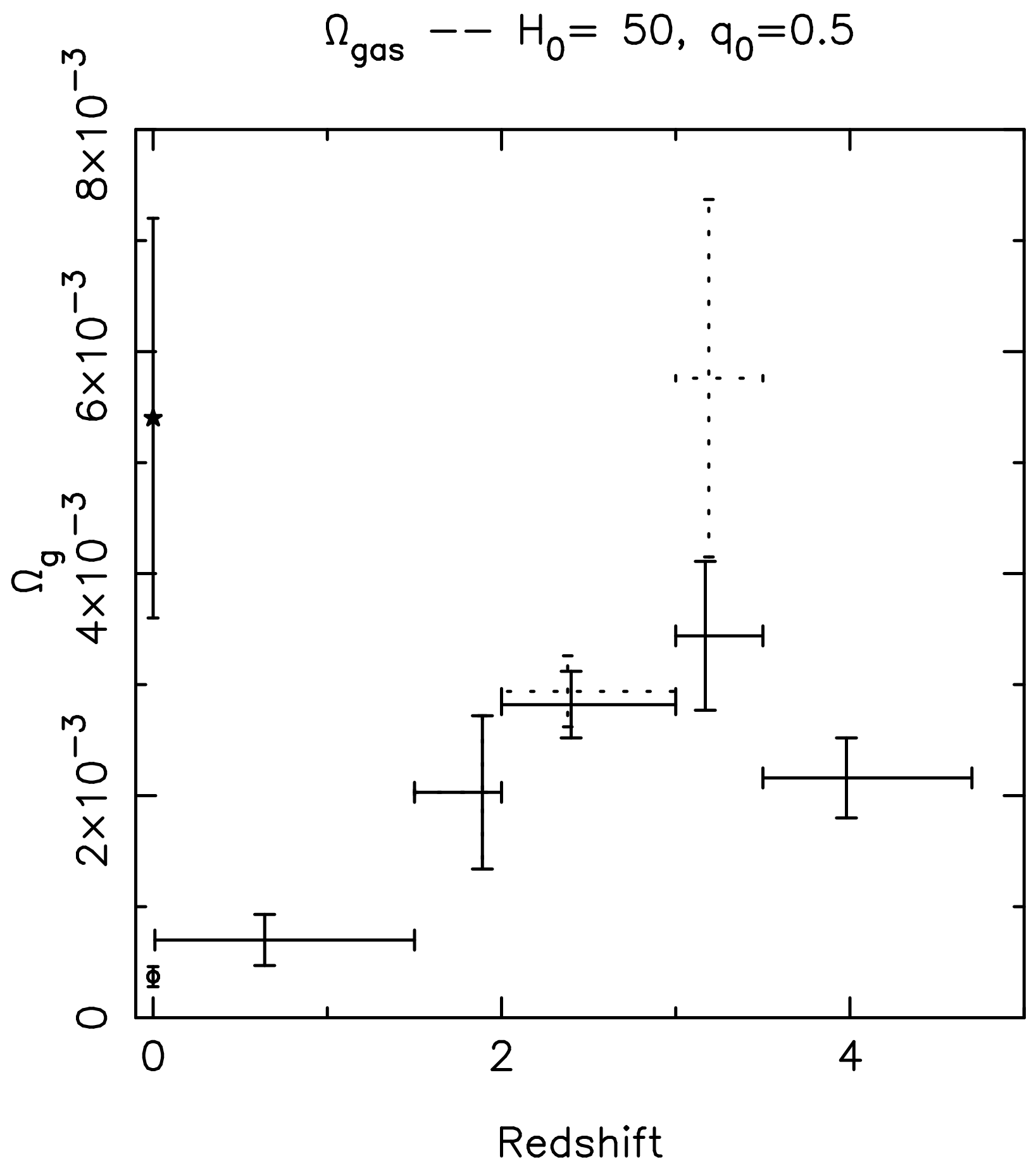

